\title{
Exploring Exercise-induced BDNF Pathways that affect Tau Dephosphorylation and Episodic Memory in Alzheimer's Disease: A Systematic Review
}

\author{
Simona Gindin, BHSc Student [1]* \\ [1] Faculty of Health Sciences, McMaster University, Hamilton, ON, Canada L8S 4L8 \\ *Corresponding Author: gindins@mcmaster.ca
}

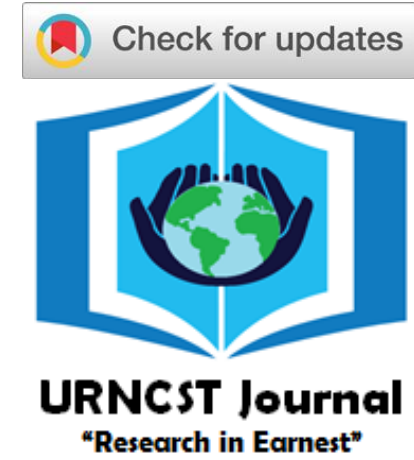

\begin{abstract}
Introduction: Research has indicated that exercise improves memory and cognitive function, which can be attributed to an increase in exercise-induced brain-derived neurotrophic factor (BDNF). BDNF has also been suggested to reverse tau aggregation seen in Alzheimer's disease (AD) via tau dephosphorylation, which can thereby improve memory function. A tyrosine receptor (TrkB) mechanism has been proposed between BDNF and the subsequent tau dephosphorylation. However, the effects of exercise-induced BDNF on tau dephosphorylation and episodic memory remain unclear. Thus, the purpose of the systemic review is to clarify a pathway linking exercise, BDNF, tau dephosphorylation, and episodic memory in AD models.

Methods: A comprehensive literature search of peer-reviewed primary and review articles was conducted in the field of neuroscience and memory. Key search terms used in the database were: BDNF, tau, phosphorylation, TrkB, exercise, memory, and Alzheimer's disease.

Results: Evidence suggests that exercise-induced BDNF enhances episodic memory in animal and human studies, and has therapeutic potential for alleviating AD symptoms. Furthermore, bath incubation of BDNF administered to AD-induced human and rodent tissue has demonstrated a rapid tau dephosphorylation effect, specifically through a protein kinase pathway involving PI-3K and AKT, following TrkB binding.

Discussion: The memory enhancements demonstrated from increased BDNF production are dependent on exercise. Exerciseinduced episodic memory enhancements may be explained by BDNF's ability to dephosphorylate tau via a TrkB mechanism. Specifically, the PI-3K/AKT pathway is the subsequent downstream signal involved in tau dephosphorylation, since BDNF administration to $\mathrm{AD}$-induced neurons resulted in no dephosphorylation in the presence of a PI-3K inhibitor. Lastly, given BDNF's ability to dephosphorylate tau in AD models and shift tau accumulation away from the soma, it indicates that exercise may form part of an effective treatment for individuals with AD.

Conclusion: Although the research surrounding BDNF and tau dephosphorylation on episodic memory enhancements is extensive, gaps remain about BDNF's effects in an exercise-induced setting. Further research needs to be conducted to confirm whether exercise-induced BDNF indicates similar effects on episodic memory. This research is also clinically relevant in $\mathrm{AD}$ treatment, whereby exercise has the potential to be prescribed concurrently with other therapies.
\end{abstract}

Keywords: exercise; BDNF; memory; tau; phosphorylation; Alzheimer's disease; TrkB

\section{Introduction}

Living an active lifestyle, through exercise and diet, has faced growing popularity given its physical, social, and emotional benefits. The scientific community has shown interest in the benefits of exercise, specifically as a therapeutic intervention due to its improvements in mood and sleep quality, and its ability to reduce the risk and progression of many diseases [1]. One particular area of research that needs to be further explored is the effect and impact of exercise on episodic memory, a form of declarative memory that is associated with the recollection of previous experiences of events [2], and whether exercise is a potentially effective treatment for neurodegenerative diseases associated with memory impairment. Extensive research has demonstrated that exercise induces neuroplasticity, the ability to form new neural connections, and can improve memory and cognitive function [3-6]. Specifically pertaining to memory, exercise-induced neuroplasticity can be a result of increased neurogenesis, cell proliferation, and dendritic branching in the hippocampus, a brain structure with a major role in learning and memory [3]. Many studies assert that aerobic exercise also increases levels of central brain-derived neurotrophic factor (BDNF) [3-5], a neurotrophin that induces neuronal survival and growth, a modulator for neuroplasticity [6], and is required for the formation of long-term memory 
UNDERGRADUATE RESEARCH IN NATURAL AND CLINICAL SCIENCE AND TECHNOLOGY (URNCST) JOURNAL Read more URNCST Journal articles and submit your own today at: https://www.urncst.com

storage [7]. Thus, exercise-induced BDNF may result in improvements in episodic memory performance due to its effect in changing synaptic plasticity [3-5].

Given the positive impact of exercise-induced BDNF on enhancing memory and cognition, exercise has therapeutic potential for treating the symptoms of Alzheimer's disease (AD). $\mathrm{AD}$ is a progressive and irreversible neurodegenerative disorder, and manifests clinically as a loss of episodic memory and a decline in cognitive function $[8,9]$. The molecular manifestation of $\mathrm{AD}$ is characterized by the loss of synaptic plasticity and synapses [8]. The neuropathology of $\mathrm{AD}$ can be explained by the formation of beta-amyloid plaques and neurofibrillary tangles (NFT) composed of aggregations of hyperphosphorylated tau protein in neurons $[8,10]$. Focusing on tau effects on $\mathrm{AD}$ pathology, the hyperphosphorylation of tau can disrupt axonal transport, mitochondrial function, and impair signalling pathways in the presynaptic and postsynaptic compartments of neurons [8]. When tau proteins spread to the hippocampus and other limbic structures, deficits in memory become more apparent, although the neurobiological mechanism underlying this change in memory remains elusive $[8,10,11]$. It has been proposed that memory deficits can arise from neurofibrillary degeneration in the basal forebrain that results in a subsequent loss of cholinergic neurons and presynaptic cholinergic innervations [8]. Alternatively, memory deficits could also manifest as a result of an accumulation of hyperphosphorylated tau in the hippocampus [12] or a combination of both proposed mechanisms occurring simultaneously. From these studies, it has been suggested that tau has a role in $\mathrm{AD}$ development and can be a therapeutic target for remediation measures.

A potential solution for mediating tau hyperphosphorylation is through the use of BDNF. Evidence suggests that lower levels of BDNF exist in AD human post-mortem tissue samples relative to tissue samples without AD pathology [13]. However, when BDNF is administered through bath incubation of tissue samples, it has been found to induce the dephosphorylation of tau in both $\mathrm{AD}$-induced human neuroblastoma cells and mouse embryonic carcinoma cells [14,15]. Although the exact mechanism between BDNF and tau is indeterminate, a tyrosine receptor kinase B (tropomyosin receptor kinase $\mathrm{B}$, TrkB) signalling pathway has been suggested [16]. As a specific receptor of BDNF, the BDNF-TrkB pathway plays an important role in cell survival, synaptic transmission, synapse remodeling, and axonal and dendritic outgrowth [17]. There is also evidence suggesting that treadmill exercise can reduce hyperphosphorylated tau in an $\mathrm{AD}$ induced rat model [18]. Thus, there is some evidence that suggests a role that exercise-induced BDNF plays in the onset and possible alleviation of $\mathrm{AD}$ symptoms.

Although there is a possibility that BDNF may improve episodic memory performance in $A D$ via dephosphorylation of tau given the current literature, what remains elusive is how exercise-induced BDNF could impact $\mathrm{AD}$ onset and symptoms. It is known that exercise increases levels of BDNF and reduces levels of hyperphosphorylated tau. Further, research has shown that bath incubation of BDNF induces a dephosphorylation of tau. However, what remains to be explored is whether exercise increasing BDNF levels produces the same effect. Thus, the purpose of this systematic review is to better define a mechanistic pathway connecting exercise, BDNF, and tau dephosphorylation that could be further explored to improve memory performance in $\mathrm{AD}$.

\section{Methods}

Upon solidification of the research question, a comprehensive search of peer-reviewed literature search was conducted in the fields of neuroscience and memory. Key terms that were searched include; BDNF, tau, Alzheimer's disease, memory, exercise, TrkB, and phosphorylation. In addition, other sources were found based on primary citations found in relevant journal articles and those that cited said journal. All journals were screened for inclusion based on date of publication (published 2004 and onward) and relevance to the research topic. All relevant information was extracted, collated, summarized, and organized into the current paper.

\section{Results}

Exercise-induced BDNF effects on episodic memory

As previously mentioned, multiple studies have shown that aerobic exercise is known to increase levels of hippocampal BDNF. A study by Neeper et al. subjected rodents to voluntary exercise on an activity wheel for 7 days, which were shown to have increased expression of BDNF messenger RNA (mRNA) in the hippocampus and in the caudal region of the neocortex [3,4]. Vaynman et al. demonstrated that rodents which exercise voluntarily performed better than sedentary controls in the Morris water maze (MWM), a behavioural task that examines spatial memory and learning $[3,19]$. Given that BDNF acts on the TrkB receptor, Vaynman et al. also aimed to demonstrate that the apparent cognitive and memory improvements seen post-exercise are dependent on exercise-induced BDNF by blocking TrkB receptors with the injection of TrkB-IgG immunoadhesin chimeras $[3,4,19]$. Rats were randomly assigned to four groups: exercising or sedentary with cytochrome $\mathrm{C}$ (cytC) control injection, and exercising or sedentary with TrkB-IgG injection [19]. Drug treatments were administered in the first week of exercise before MWM training [19]. The marked improvements in spatial memory at encoding and test in the exercising/cytC group were suppressed in the exercising/TrkB group $[3,4,19]$. However, blocking BDNF in sedentary controls resulted in no change in BDNF mRNA levels and had no effect on episodic memory [3,19]. Multiple human studies have also demonstrated that levels of serum/plasma BDNF were enhanced following exercise 
UNDERGRADUATE RESEARCH IN NATURAL AND CLINICAL SCIENCE AND TECHNOLOGY (URNCST) JOURNAL Read more URNCST Journal articles and submit your own today at: https://www.urncst.com

in healthy individuals [4]. A study by Rasmussen et al. measured BDNF levels following ergometer rowing that were secreted from the brain using a catheter [4]. They found a four-fold increase in BDNF levels after four hours of exercise compared to levels at rest [4]. As well, Griffin et al. examined the effects of exercise on the performance of working memory through a face-name matching task, known to recruit the hippocampus, compared to the Stroop word-colour task which does not recruit the hippocampus $[4,20]$. They found that following high-intensity cycling, improvements were seen in the face-name matching task, as well as elevated serum BDNF levels, but not in the Stroop word-colour task [4,20]. Thus, exercise-induced BDNF has promising effects on enhancing episodic memory in both animal and human models.

\section{BDNF's effects on tau dephosphorylation}

Evidence suggests that BDNF may result in tau dephosphorylation in an animal model of AD. A study by Elliott et al. used neuronally differentiated post-natal day 19 (P19) mouse embryonic carcinoma cells and found that upon BDNF bath incubation, there was a rapid dephosphorylation of tau at all phosphorylation sites except for site Thr181 [15]. To validate BDNF's role in tau dephosphorylation, a Trk inhibitor, K252a, was administered and decreased the dephosphorylation of tau induced by BDNF [15].

Another study demonstrated that in undifferentiated human SH-SY5Y neuroblastoma cells lacking neurites, retinoic acid (RA) treatment increased levels of tau and decreased tau phosphorylation at Ser262, as well as induced neurite outgrowth [14]. After a subsequent treatment of BDNF, these effects were further augmented [14]. They also found that in undifferentiated cells, tau accumulated in the soma and that the spherical structure of the soma in undifferentiated cells resembled tau aggregations in $\mathrm{AD}$ [14]. Upon RA and BDNF treatment, it was observed that neurite outgrowth increased and tau shifted from the soma to the neurites [14].

Given the complexity of the underlying mechanism of $\mathrm{AD}$ pathology, Bharani et al. aimed to assess the role of BDNF in AD by examining the relationship between BDNF levels, phosphorylated tau, and BDNF receptor density [13]. They found that serum pro-BDNF was negatively correlated with hippocampal pro-BDNF, but was positively correlated with phosphorylated tau (percent area) in postmortem brain samples from individuals with severe AD [13]. Percent area of TrkB was also negatively correlated with phosphorylated tau measurements [13]. Thus, BDNF has demonstrated to be associated with tau dephosphorylation in AD-induced models via a TrkB mechanism.

\section{BDNF-induced tau dephosphorylation through a TrkB} mechanism

The purpose of Elliott et al.'s study was to examine the relationship between BDNF and tau phosphorylation, as well as to distinguish which signaling pathway is responsible for tau phosphorylation upon BDNF-TrkB binding [15]. Elliott et al. proposed that the effect BDNF has on tau phosphorylation is through TrkB receptor signalling [15]. Within the TrkB mechanism there may be three potential pathways (Figure 1) that could be implicated during BDNF-induced tau dephosphorylation; the phosphatidylinositol 3-kinase/protein kinase B (PI$3 \mathrm{~K} / \mathrm{AKT}$ ) pathway, the mitogen-activated protein kinase/extracellular-signal-regulated kinase (MAPK/ERK) pathway, or the phospholipase C- $\gamma /$ protein kinase C (PLC $\gamma$ / PKC) pathway [21]. These pathways typically regulate axonal and dendritic growth, neuronal proliferation and differentiation, and synaptic plasticity, respectively, as well as all three pathways contributing to neuronal survival [21].

In terms of identifying the role of the MAPK/ERK and the PLC $\gamma /$ PKC pathways in tau dephosphorylation, Elliott et al. concluded that the observed tau dephosphorylation by BDNF is independent of both pathways [15]. To rule out the MAPK/ERK pathway, P19 neurons were pretreated with PD98059, a MAPK inhibitor, and then were incubated with or without BDNF [15]. They found that PD98059 alone slightly reduced tau phosphorylation, while neurons with BDNF increased reactivity of Tau1, an antibody associated with levels of non-phosphorylated tau, and decreased reactivity of AT8, an antibody associated with levels of phosphorylated tau, when compared to neurons without BDNF incubation [15]. To rule out the PLC $\gamma / \mathrm{PKC}$ pathway, P19 neurons were incubated with GF109203X, a PKC inhibitor [15]. Upon administration of BDNF, tau dephosphorylation occurred even in the presence of GF109203X [15]. Thus, BDNF-induced tau dephosphorylation is not dependent on the MAPK/ERK or the PLC $\gamma /$ PKC pathways.

In terms of identifying the role of the PI-3K/AKT pathway in tau dephosphorylation, evidence suggests that tau dephosphorylation from BDNF is dependent on this pathway $[15,21,22]$. When this pathway is activated, PI-3K activates AKT through phosphorylation, which then subsequently phosphorylates and inhibits glycogen synthase kinase $3 \beta$ (GSK-3 $\beta$ ), a kinase that contributes to tau phosphorylation [21]. Elliott et al. confirmed that BDNF acts on PI-3K given that treatment of P19 neurons with wortmannin, a PI-3K inhibitor, inhibited the phosphorylation of AKT and a subsequent stimulation with BDNF resulted in no change in either Tau1 or AT8 antibody levels [15]. As well, pre-treatment with lithium, a GSK-3 $\beta$ inhibitor, without BDNF resulted in increased reactivity of the Tau 1 antibody and decreased reactivity of the AT8 antibody, which both correspond to tau dephosphorylation [15]. However, when P19 neurons were incubated with lithium and then either with BDNF or without BDNF, there was no difference in Tau1 or AT8 reactivity between the two groups [15]. They concluded that when GSK-3 $\beta$ is inhibited prior to BDNF administration, BDNF is unable to reduce phosphorylation levels of tau [15]. Thus, upon binding to TrkB, BDNF- 
UNDERGRADUATE RESEARCH IN NATURAL AND CLINICAL SCIENCE AND TECHNOLOGY (URNCST) JOURNAL Read more URNCST Journal articles and submit your own today at: https://www.urnest.com

induced tau dephosphorylation is dependent on the inhibition of GSK-3 $\beta$, which is mediated through the PI$3 \mathrm{~K} / \mathrm{AKT}$ pathway [15].

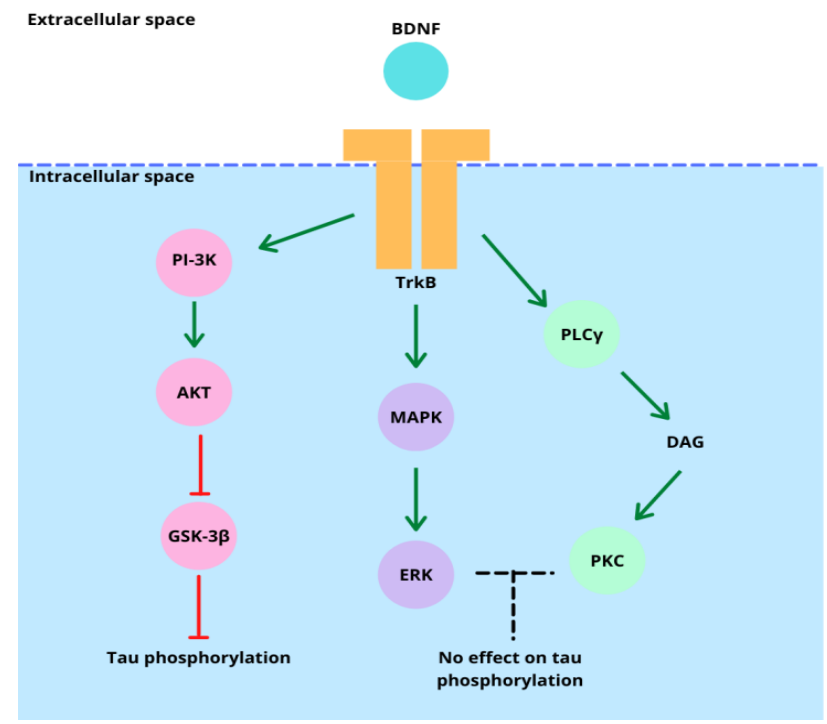

Figure 1. Downstream signalling cascade of BDNF binding to TrkB.

Three predominant downstream pathways exist in the BDNF-TrkB mechanism. Pink: The PI-3K/AKT pathway phosphorylates GSK-3 $\beta$, inhibiting its phosphorylation of tau in the process. Purple and Green: The MAPK/ERK and PLC $\gamma$ / PKC pathway, respectively, have no apparent role in tau dephosphorylation. Green arrows indicate activation, red line indicates inhibition, blue dotted line indicates the cell membrane.

\section{Discussion}

BDNF is a promising therapeutic agent for the remediation of $\mathrm{AD}$ neuropathology and symptoms. A current gap in the research exists whereby the effects of BDNF, specifically produced from exercise, on tau dephosphorylation remain elusive. Thus, the purpose of the review was to aggregate the aforementioned results and outline a pathway that defines how exercise-induced increases in BDNF can affect episodic memory and potential clinical implications in AD.

Firstly, analyzing how exercise-induced BDNF affects memory retention in rodents, Neeper et al. suggested an association of BDNF with memory-dependent brain regions. They described that exercise could increase levels of BDNF in the hippocampus and the neocortex [4]. Vaynman et al.'s study demonstrated that exercising rodents that were injected with a TrkB inhibitor had suppressed spatial memory acquisition and retention enhancements that were seen in their non-inhibitor counterparts $[4,19]$. This finding helps to solidify BDNF's downstream effects on memory retention via BDNFspecific TrkB receptor pathways. Since improvements in memory retention were seen after exercise, and a subsequent suppression of memory performance was seen following receptor inhibition, this indicates that exerciseinduced BDNF is necessary and sufficient to modulate memory function through the TrkB receptor in rodent models. Injection of a TrkB inhibitor in sedentary controls resulted in no change in memory retention, demonstrating that the enhancements in memory retention are dependent on exercise, not merely endogenous-BDNF present at baseline levels $[4,19]$. Thus, there must be an increase in BDNF through exercise in order to see improvements in spatial memory. Griffin et al.'s findings further support that exercise-induced BDNF improves episodic memory, given that improved performance was found in a hippocampaldependent face-name matching task following exercise compared to no improvements in the Stroop word-colour task, a task independent of hippocampal function [4]. Since these results isolate for a memory-dependent region, it demonstrates that this induced increase in BDNF specifically affects memory processes. Altogether, these findings suggest that exercise-induced BDNF can enhance episodic memory, including instances where there is memory impairment, like in $\mathrm{AD}$. However, what remains to be understood is the underlying mechanism by which this can occur.

The underlying mechanism by which BDNF improves memory in $\mathrm{AD}$ models can be addressed when further analyzing how BDNF and tau are correlated, with BDNF playing an important role in tau dephosphorylation. Since Griffin et. al indicated that serum levels of BDNF were elevated post-exercise and demonstrated improved hippocampal function, BDNF may modulate levels of tau aggregates that also accumulate in the hippocampus in $\mathrm{AD}$ pathology [4]. In fact, Elliott et al. demonstrated a dephosphorylation effect upon bath incubation of BDNF and then a reversed effect upon TrkB inhibition, concluding that BDNF has downstream effects on tau phosphorylation [15]. It can also be deduced that BDNF would dephosphorylate tau in $\mathrm{AD}$ models given that treatment of $\mathrm{BDNF}$ in human AD-induced neuroblastoma cells resulted in decreased tau phosphorylation at Ser262 [14]. The finding that BDNF resulted in neurite outgrowth and the shift of tau away from the soma can possibly be explained by decreased tau phosphorylation [14]. This effect may potentially help alleviate further decline in $\mathrm{AD}$ given that AD pathology involves an accumulation of tau in the soma [14]. Although this study used bath incubated BDNF, it can be suggested that exercise-induced BDNF could have similar effects in in vivo $\mathrm{AD}$ models after treatment. As well, Bharani et al.'s study provided additional evidence for the correlation between high levels of BDNF in the hippocampus and lower levels of phosphorylated tau in individuals with $\mathrm{AD}$, although this was only experimented with pro-BDNF [13]. They also demonstrated that BDNF affects tau phosphorylation through a TrkB mechanism given the negative correlation between TrkB area and 
UNDERGRADUATE RESEARCH IN NATURAL AND CLINICAL SCIENCE AND TECHNOLOGY (URNCST) JOURNAL Read more URNCST Journal articles and submit your own today at: https://www.urncst.com

phosphorylated tau. Thus, the pathway involving endogenous BDNF and tau has been suggested given these findings in the literature: that exercise-induced BDNF may induce tau dephosphorylation through a TrkB-dependent mechanism.

Elliott et al. helps to further explain the next step in the BDNF-tau phosphorylation mechanism by investigating the activity beyond BDNF binding to the TrkB receptor [15]. Given that administration of BDNF resulted in tau dephosphorylation despite mouse embryonic P19 carcinoma neurons being treated with either the MAPK/ERK inhibitor or the PLC $\gamma$ / PKC inhibitor, it indicates that, during tau dephosphorylation, BDNF does not operate via the MAPK/ERK or the PLC $\gamma / \mathrm{PKC}$ pathway [15]. However, since administration of BDNF in the presence of wortmannin resulted in no change in tau antibody levels (no dephosphorylation was observed), BDNF's effects on tau phosphorylation is dependent on the PI-3K/AKT pathway [15]. Furthermore, since Tau1 reactivity increased and AT8 reactivity decreased, a tau dephosphorylation effect was seen with lithium [15]. Although administration of BDNF with lithium resulted in no change in antibody reactivity, lithium acts as a positive control, demonstrating that BDNF also inhibits GSK-3 $\beta$. Since a dephosphorylation effect was seen with BDNF stimulation on P19 neurons and lithium, it can be confirmed that BDNF operates through the PI-3K/AKT pathway with a downstream target on GSK-3 $\beta$ when modulating levels of tau [15]. These findings coincide with Wang et al.'s suggestion that inhibition of GSK-3 $\beta$ is one of the ways in which tau dephosphorylation may be induced in AD models [23]. Although the current literature has helped to define BDNF's actions on TrkB through a PI-3K/AKT and GSK$3 \beta$ pathway, further downstream effects are still unknown as the extent of research has not reached this point and the underlying mechanism remains elusive.

While the current findings help to solidify the link between the exercise, BDNF, tau dephosphorylation, and its effects on episodic memory performance, a missing component persists throughout each study. The studies that demonstrate exercise increasing BDNF and enhancing episodic memory performance lack the tau dephosphorylation component. The studies that demonstrate BDNF inducing tau dephosphorylation have not yet explored the effects from an increase of BDNF via exercise. The studies that indicate the effects of BDNF on TrkB through a PI-3K/AKT mechanism and resulting in tau dephosphorylation also lack information on exercisedinduced BDNF as a receptor ligand. Thus, based on the present findings, future studies should explore how exercise-induced BDNF affects tau dephosphorylation and subsequent impacts on episodic memory. Future research may reveal that BDNF levels will increase in exercising AD-induced rodent models, which will then act on TrkB and cause a dephosphorylation of tau in the hippocampus through a PI-3K/AKT pathway, and thus demonstrate potential for exercise-induced BDNF to alleviate early $\mathrm{AD}$ pathology and to enhance performance in episodic memory.

\section{Conclusion}

Based on the conclusions that exogenous BDNF modulates tau phosphorylation through the TrkB and PI3K/AKT pathway, exercise-induced BDNF may also have an effect on tau dephosphorylation through this mechanism and positively affect episodic memory. It is understood that exercise-induced BDNF aids in the enhancement of episodic memory performance. Furthermore, bath incubation of BDNF was seen to induce dephosphorylation of tau in $\mathrm{AD}$ mouse and human tissue models. Lastly, incubated BDNF acts on the TrkB receptor solely through the PI-3K/AKT pathway, however BDNF's actions past GSK-3 $\beta$ remain elusive. As outlined, gaps remain in the research in terms of exercise-induced BDNF's effects on tau dephosphorylation and episodic memory. Although the apparent tau dephosphorylation pathway could only be examined with rodent models, further research is required in order to apply these conclusions at a clinical level. In addition, further research needs to be divulged to confirm that endogenous BDNF, increased because of exercise, also dephosphorylates tau through the PI-3K/AKT pathway, resulting in improved episodic memory. Given that much of the research has explored tau phosphorylation in $\mathrm{AD}$ models, the research surrounding exercise's effects on the enhancement of episodic memory can be applied to the AD population. Potentially, the effects of exercise on episodic memory can be an avenue for future research in the prevention and delayed progression of $\mathrm{AD}$ in its early stages and may have potential to act concurrently with other pharmacological therapies for AD.

\section{List of Abbreviations Used}

BDNF: brain-derived neurotrophic factor

AD: Alzheimer's disease

TrkB: tyrosine receptor kinase B (tropomyosin receptor kinase B)

PI-3K: phosphatidylinositol 3-kinase

AKT: protein kinase B

MWM: morris water maze

cytC: cytochrome $\mathrm{C}$

MAPK: mitogen-activated protein kinase

ERK: extracellular-signal-regulated kinase

PLC $\gamma$ : phospholipase C- $\gamma$

PKC: protein kinase $\mathrm{C}$

GSK-3 $\beta$ : glycogen synthase kinase $3 \beta$

\section{Conflicts of Interest}

I declare that there are no conflicts of interest.

Ethics Approval and/or Participant Consent

As this study was a systematic peer review, ethics approval was not required. 
UNDERGRADUATE RESEARCH IN NATURAL AND CLINICAL SCIENCE AND TECHNOLOGY (URNCST) JOURNAL Read more URNCST Journal articles and submit your own today at: https://www.urncst.com

\section{Authors' Contributions}

SG: made contributions to the design of the study, collected and analysed data, drafted the manuscript, and gave final approval of the version to be published.

\section{Acknowledgements}

I would like to acknowledge my mentor, Bi-ru Amy Yeung, for guiding me through the research, writing process, and making contributions to editing the systematic review.

\section{Funding}

This study was not funded.

\section{References}

[1] Altmann LJP, Stegemöller E, Hazamy AA, Wilson JP, Bowers D, Okun MS, et al. Aerobic exercise improves mood, cognition, and language function in Parkinson's disease: Results of a controlled study. J Int Neuropsychol Soc JINS. 2016;22(9):878-89. https://doi.org/10.1017/S135561771600076X

[2] Tulving E, Markowitsch HJ. Episodic and declarative memory: Role of the hippocampus. Hippocampus. 1998;8(3):198-204. https://doi.org/10.1002/(SICI)10981063(1998)8:3<198::AID-HIPO2>3.0.CO;2-G

[3] Cassilhas RC, Tufik S, de Mello MT. Physical exercise, neuroplasticity, spatial learning and memory. Cell Mol Life Sci. 2016 Mar;73(5):975-83. https://doi.org/10.1007/s00018-015-2102-0

[4] Wang R, Holsinger RMD. Exercise-induced brainderived neurotrophic factor expression: Therapeutic implications for Alzheimer's dementia. Ageing Res Rev. 2018 Dec;48:109-21. https://doi.org/10.1016/ j. arr.2018.10.002

[5] Hayek LE, Khalifeh M, Zibara V, Assaad RA, Emmanuel N, Karnib N, et al. Lactate mediates the effects of exercise on learning and memory through SIRT1-dependent activation of hippocampal brainderived neurotrophic factor (BDNF). J Neurosci. 2019 Mar 27;39(13):2369-82. https://doi.org/10.1523/ JNEUROSCI.1661-18.2019

[6] Bathina S, Das UN. Brain-derived neurotrophic factor and its clinical implications. Arch Med Sci. 2015 Dec 10;11(6):1164-78. https://doi.org/10.5114/aoms.2015 .56342

[7] Bekinschtein P, Cammarota M, Izquierdo I, Medina JH. BDNF and memory formation and storage. Neuroscientist. 2008 Apr;14(2):147-56. https://doi.org/ $\underline{10.1177 / 1073858407305850}$

[8] Soria Lopez JA, González HM, Léger GC. Chapter 13 - Alzheimer's disease. In: Dekosky ST, Asthana S, editors. Handbook of clinical neurology [Internet]. Elsevier; 2019 [cited 2021 Feb 19]. p. 231-55. (Geriatric Neurology; vol. 167). Available from:

Gindin | URNCST Journal (2021): Volume 5, Issue 6 DOI Link: https://doi.org/10.26685/urncst.265
https://www.sciencedirect.com/science/article/pii/B978 0128047668000133

[9] Silva MVF, Loures C de MG, Alves LCV, de Souza LC, Borges KBG, Carvalho M das G. Alzheimer's disease: Risk factors and potentially protective measures. J Biomed Sci. 2019 May 9;26(1):33. https://doi.org/10.1186/s12929-019-0524-y

[10] Jahn H. Memory loss in Alzheimer's disease. Dialogues Clin Neurosci. 2013 Dec;15(4):445-54. https://doi.org/10.31887/DCNS.2013.15.4/hjahn

[11] Biundo F, Del Prete D, Zhang H, Arancio O, D’Adamio L. A role for tau in learning, memory and synaptic plasticity. Scientific Reports. 2018 Feb 16;8(1):3184. https://doi.org/10.1038/s41598-018-21596-3

[12] Harrison TM, Maass A, Adams JN, Du R, Baker SL, Jagust WJ. Tau deposition is associated with functional isolation of the hippocampus in aging. Nat Commun. 2019 Oct 25;10(1):4900. https://doi.org/10.1038/s41467019-12921-Z

[13] Bharani KL, Ledreux A, Gilmore A, Carroll SL, Granholm A-C. Serum pro-BDNF levels correlate with phospho-tau staining in Alzheimer's disease. Neurobiol Aging. 2020 Mar;87:49-59. https://doi.org/10.1016/ j.neurobiolaging.2019.11.010

[14] Chen Q, Zhou Z, Zhang L, Xu S, Chen C, Yu Z. The cellular distribution and Ser262 phosphorylation of tau protein are regulated by BDNF in vitro. PLoS One. 2014;9(3):e91793. https://doi.org/10.1371/journal.pone .0091793

[15] Elliott E, Atlas R, Lange A, Ginzburg I. Brain-derived neurotrophic factor induces a rapid dephosphorylation of tau protein through a PI-3Kinase signalling mechanism. European Journal of Neuroscience. 2005;22(5):1081-9. https://doi.org/10.1111/j.14609568.2005.04290.x

[16] Zhang F, Kang Z, Li W, Xiao Z, Zhou X. Roles of brainderived neurotrophic factor/tropomyosin-related kinase $\mathrm{B}$ (BDNF/TrkB) signalling in Alzheimer's disease. Journal of Clinical Neuroscience. 2012 Jul 1;19(7):946-9. https://doi.org/10.1016/j.jocn.2011.12.022

[17] Ohira K, Hayashi M. A new aspect of the trkb signaling pathway in neural plasticity. Curr Neuropharmacol. 2009 Dec;7(4):276-85. https://doi.org/10.2174/157015909790031210

[18] Lu Y, Dong Y, Tucker D, Wang R, Ahmed ME, Brann $\mathrm{D}$, et al. Treadmill exercise exerts neuroprotection and regulates microglial polarization and oxidative stress in a streptozotocin-induced rat model of sporadic Alzheimer's disease. Journal of Alzheimer's Disease. 2017 Jan 1;56(4):1469-84. https://doi.org/10.3233/JAD160869

[19] Vaynman S, Ying Z, Gomez-Pinilla F. Hippocampal BDNF mediates the efficacy of exercise on synaptic plasticity and cognition. European Journal of Neuroscience. 2004;20(10):2580-90. https://doi.org/ 10.1111/j.1460-9568.2004.03720.x 
UNDERGRADUATE RESEARCH IN NATURAL AND CLINICAL SCIENCE AND TECHNOLOGY (URNCST) JOURNAL Read more URNCST Journal articles and submit your own today at: https://www.urncst.com

[20] Griffin ÉW, Mullally S, Foley C, Warmington SA, O’Mara SM, Kelly ÁM. Aerobic exercise improves hippocampal function and increases BDNF in the serum of young adult males. Physiology \& Behavior. 2011 Oct 24;104(5):934-41. https://doi.org/10.1016/ j.physbeh.2011.06.005

[21] Tejeda GS, Díaz-Guerra M. Integral characterization of defective BDNF/TrkB signalling in neurological and psychiatric disorders leads the way to new therapies. International Journal of Molecular Sciences. 2017 Feb;18(2):268. https://doi.org/10.3390/ijms18020268
[22]Zhang F, Kang Z, Li W, Xiao Z, Zhou X. Roles of brain-derived neurotrophic factor/tropomyosin-related kinase B (BDNF/TrkB) signalling in Alzheimer's disease. J Clin Neurosci. 2012 Jul;19(7):946-9. https://doi.org/10.1016/j.jocn.2011.12.022

[23] Wang JZ, Grundke-Iqbal I, Iqbal K. Kinases and phosphatases and tau sites involved in Alzheimer neurofibrillary degeneration. Eur J Neurosci. 2007 Jan;25(1):59-68. https://doi.org/10.1111/j.1460-9568 $.2006 .05226 . x$

\section{Article Information}

Managing Editor: Jeremy Y. Ng

Peer Reviewers: Bi-ru Amy Yeung, Ricky Chow

Article Dates: Received Apr 08 21; Accepted May 18 21; Published Jun 0921

\section{Citation}

Please cite this article as follows:

Gindin S. Exploring exercise-induced BDNF pathways that affect tau dephosphorylation and episodic memory in Alzheimer's disease: A systematic review. URNCST Journal. 2021 Jun 09: 5(6).

https://urncst.com/index.php/urncst/article/view/265

DOI Link: https://doi.org/10.26685/urncst.265

\section{Copyright}

(c) Simona Gindin. (2021). Published first in the Undergraduate Research in Natural and Clinical Science and Technology (URNCST) Journal. This is an open access article distributed under the terms of the Creative Commons Attribution License (https://creativecommons.org/licenses/by/4.0/), which permits unrestricted use, distribution, and reproduction in any medium, provided the original work, first published in the Undergraduate Research in Natural and Clinical Science and Technology (URNCST) Journal, is properly cited. The complete bibliographic information, a link to the original publication on http://www.urncst.com, as well as this copyright and license information must be included.

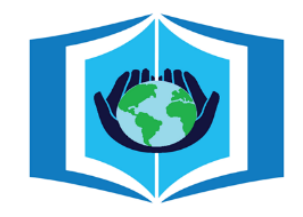

\section{URNCST Journal \\ "Research in Earnest"}

\section{Funded by the Government of Canada}

\section{Canadà}

Do you research in earnest? Submit your next undergraduate research article to the URNCST Journal!

| Open Access | Peer-Reviewed | Rapid Turnaround Time | International |

| Broad and Multidisciplinary | Indexed | Innovative | Social Media Promoted |

Pre-submission inquiries? Send us an email at info@ urncst.com | Facebook, Twitter and LinkedIn: @ URNCST

Submit YOUR manuscript today at https://www.urnest.com! 